\title{
Molecular Reaction Imaged by Scanning Atom Probe*
}

\author{
Masahiro Taniguchi ${ }^{\dagger}$ and Osamu Nishikawa \\ Kanazawa Institute of Technology, 7-1 Ohgigaoka, Nonoichi, Ishikawa 921-8501, Japan \\ (Received 8 January 2010; Accepted 20 January 2010; Published 13 February 2010)
}

\begin{abstract}
Organic molecule system supported on metal substrate was analyzed by atom probe with two-dimensional ion detector and scanning extractor electrode (3D-SAP). The comparison of angular distribution of detected ions between molecular system and common metal tip showed a significant difference when AP analysis was performed by voltage pulsing. The influence of catalytic property of metal substrate also demonstrated by changing the substrate material. [DOI: 10.1380/ejssnt.2010.69]
\end{abstract}

Keywords: Scanning atom probe; Field ionization; Mass spectroscopy; Titanium; Tungsten; Aromatics

\section{INTRODUCTION}

Atom probe (AP) is known as the one of the most sensitive technique for solid material analysis due to its extremely high detection efficiency of elements. The atomby-atom field evaporation of apex the sample tip enables us to obtain the depth profile of the material in atomic scale by AP analysis [1]. The combination of pulsed laser and two dimensional position sensitive detector to AP realized the three dimensional tomographic analysis of both metallic and non-metallic materials in nano-meter scale (3D-AP, laser assisted AP) [2].

The authors report here the application of AP on the molecular based system supported on needle shaped specimen. In this system, the sharp tip is serving as the solid support and the field generator for field ionization of the small molecules. Generally, the larger surface energy of the small curvature of the tip apex compared to that of the flat surface inhibits sticking of modifying material in the tip apex area.

$\mathrm{AP}$ analysis is commonly performed in cryogenic temperature $(<100 \mathrm{~K})$ to suppress the surface atom migration. The migration and diffusion are not favorable in the application of AP for the solid material in the view of $3 \mathrm{D}$ elemental analyses in nano-scale. If the sample is not cooled down enough, the migration/diffusion of the atom might cause some deviation of chemical composition and spatial distribution of elements.

In the system described here, the specimen is not cooled down therefore the surface species migrate from tip shank region to the tip apex to ionize and to desorb from the tip. The ionization of molecules is often accompanied by the bond cleavage and/or the fragmentation. Thus, the AP analysis of molecular system corresponds to monitoring of the decomposition reaction on surface.

Position sensitive detector was also used in the analyses described below. But the interpretation of the experimental data is different from those in common 3D-AP analyses of solid materials. In molecular system, the hit position of the desorbed ions on the detector is related not directly

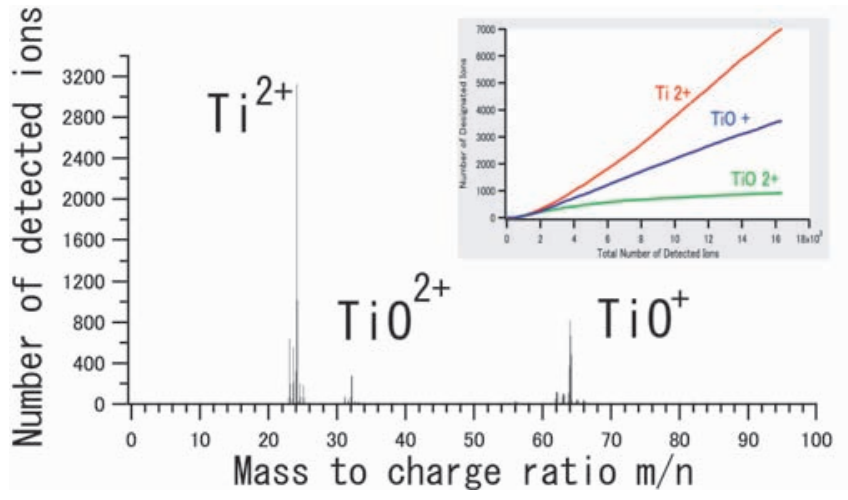

FIG. 1: The mass spectrum of Ti tip measured by laser-SAP using 2nd harmonics of Nd:YVO 4 laser. The inset shows the ladder diagram of $\mathrm{Ti}^{2+}$ (top), $\mathrm{TiO}^{+}$(middle) and $\mathrm{TiO}^{2+}$ ions (bottom).

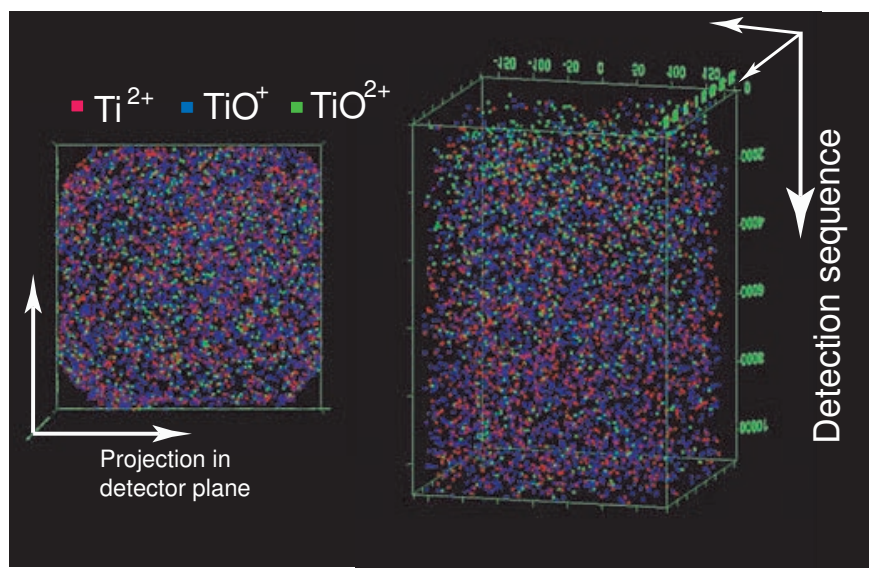

FIG. 2: Right:the distribution of $\mathrm{Ti}^{2+}$ (red), $\mathrm{TiO}^{+}$(blue) and $\mathrm{TiO}^{2+}$ ions (green) detected from Ti tip by using 3D-SAP triggered by 2nd harmonics of $\mathrm{Nd}: \mathrm{YVO}_{4}$. Left:the same data was viewed by projection in X-Y plane of detector. The colors of ions (dots) correspond to the designated mass peak and the ladder diagram of Fig. 1. The raw $(x, y, z)$ data are used for 3D plot coordinates here.
${ }^{*}$ This paper was presented at 7 th International Symposium on Atomic Level Characterizations for New Materials and Devices, The Westin Maui Resort \& Spa, Hawaii, U.S.A., 6-11 December, 2009. †Corresponding author: taniguchi@neptune.kanazawa-it.ac.jp to the spatial coordinates of the atom inside the material but simply to the angular distribution of the ionsrelated to the ionization site on the tip apex. 


\section{EXPERIMENT}

A cationic dye molecule, chloride form salt of Crystal Violet $\mathrm{C}_{25} \mathrm{~N}_{3} \mathrm{H}_{20}^{+}(\mathrm{CV}, \mathrm{M} . \mathrm{W}$. $=372.2)$, was used as probe molecule. The structure of CV is depicted in Fig. 3 as an inset. $\mathrm{CV}$ has absorption band in visible region $\left(\lambda_{\max } \approx 530 \mathrm{~nm}\right)$ close to the wavelength of the 2 nd harmonics of $\mathrm{Nd}: \mathrm{YVO}_{4}$ laser $(\lambda=532 \mathrm{~nm})$. The coating layer was prepared by dipping the substrate tip into methanol solution of CV salt and dried in the atmosphere. Electro polished titanium or tungsten tip was used as substrate.

A scanning atom probe (SAP) developed by Nishikawa et al. was used in this study. A funnel-shaped microextraction electrode confines the high electric field into a small space between a tip apex and an open end of the electrode and relaxes the severe requirement of the sample dimension $[3,4]$.

For the angular distribution analyses, SAP with two dimensional position sensitive detector (3D-SAP) was used. In this 3D-SAP system, the flight length of ions was $300 \mathrm{~mm}$ and the detection area was $40 \mathrm{~mm} \times 40 \mathrm{~mm}$. The solid angle of this system estimated in the scheme of gnomon projection was about 0.018 steradian, which was much smaller than that of modern $3 \mathrm{D}$-AP system design $(\leq 0.75$ steradian $)$.

The voltage pulser $(\mathrm{HWFM} \sim 5 \mathrm{~ns})$ or $\mathrm{Nd}: \mathrm{YVO}_{4}$ pulsed laser of pulse duration of 20ps (Ekspla PL2210JE) was used for triggering the field evaporation. The power of the laser was $0.15 \mathrm{~mJ} /$ shot for $3 \mathrm{rd}$. harmonics $(\lambda=355 \mathrm{~nm})$ and $0.2 \mathrm{~mJ} /$ shot for $2 \mathrm{nd}$. harmonics $(\lambda=532 \mathrm{~nm})$. The laser beam was focused to the spot size of $\phi=0.1 \mathrm{~mm}$ and the intensity was attenuated by using half wave plate and polarizer. The laser was operated at the repetition rate of $1000 \mathrm{~Hz}(1000 \mathrm{shot} / \mathrm{sec})$.

In the preliminary analyses with high mass resolution, SAP with a reflectron mass spectrometer (1D-SAP) whose mass resolution $\mathrm{m} / \Delta \mathrm{m}$ was better than 1000 was used. The structure of the SAP with a reflectron mass spectrometer and the fabrication process of the extraction electrode are described elsewhere [3, 4].

The raw $(\mathrm{x}, \mathrm{y}, \mathrm{z})$ data set is used for $3 \mathrm{D}$ plot without transformation for the visualization of ion distribution in this paper. The lateral hit position on the 2-dimensional detector is used for $\mathrm{X}$ and $\mathrm{Y}$ coordinates and the detection order of the each ion is used for $\mathrm{Z}$ axis position.

\section{RESULTS AND DISCUSSION}

Figure 1 shows the mass spectrum of titanium tip measured by $3 \mathrm{D}-\mathrm{SAP}$ triggered by 2 nd harmonics of $\mathrm{Nd}: \mathrm{YVO}_{4}$ laser $(\lambda=532 \mathrm{~nm})$. The peak-peak separation of isotope peaks of $\mathrm{Ti}^{2+}$ and $\mathrm{TiO}^{2+}$ are both $0.5 \mathrm{amu}$ reflecting their doubly charged state. The mass spectrum and the depth profile of ion indicate that the topmost layer of Ti tip is partially oxidized because the analysis is performed without flashing pre-treatment under UHV condition.

The right part of Fig. 2 illustrates a typical distribution of metal ions when metallic specimen is analyzed by $3 \mathrm{D}$ AP. The detection sequence proceeds from top to bottom of the right figure. The lateral coordinates recorded by 2D-detector are used for $\mathrm{X}, \mathrm{Y}$ coordinates and the detec-

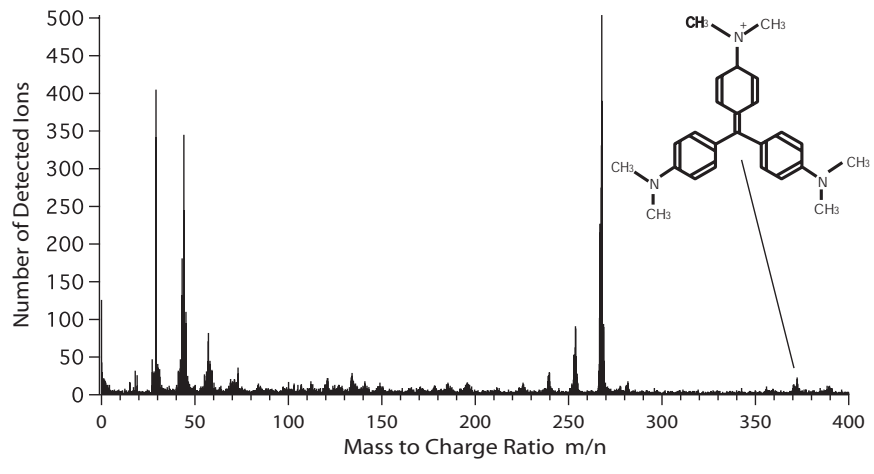

FIG. 3: The mass spectrum of CV ions detected from CV supported on Ti tip by using 3D-SAP triggered by 3rd harmonics of $\mathrm{Nd}: \mathrm{YVO}_{4}$ laser $(\lambda=355 \mathrm{~nm})$. The inset is a molecular structure of $\mathrm{CV}$ ion.

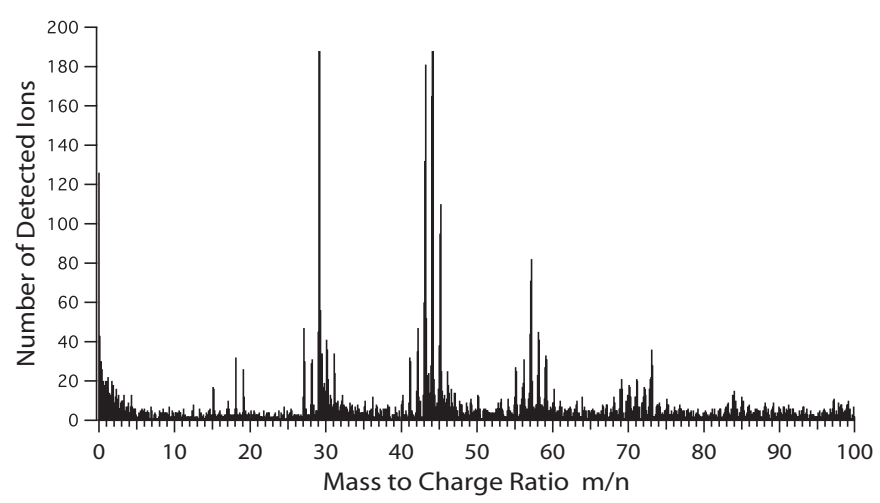

FIG. 4: The mass spectrum of CV ions detected from CV supported on Ti tip by using 3D-SAP triggered by 3rd harmonics of $\mathrm{Nd}: \mathrm{YVO}_{4}$. The Y-axis (ion count) is truncated at 200cnt for magnification.

tion order is used for $\mathrm{Z}$ (vertical)-axis coordinate in Fig. 2 without transformation to show the contrast between the angular distributions obtained for a common metal material like titanium and for molecules supported on metal substrate. In the left figure of Fig. 2, the same data is projected in $\mathrm{X}-\mathrm{Y}$ plane to show that the ions are covering the whole area of the detector and the ion density is almost homogeneous in the detection area of $40 \mathrm{~mm} \times 40 \mathrm{~mm}$. This broad and flat distribution of metal ions is generally observed also in the same way when the field evaporation is triggered by the application of voltage pulse.

Figure 3 shows the mass spectra of whole mass range (0 400 amu) obtained for CV molecule supported on Ti tip. The molecular ion peak of $\mathrm{CV}$ is observed around 372 amu. The mass spectrum shows the large fragment ions of CV $(>200 \mathrm{amu})$ and ions of smaller mass $(<100 \mathrm{amu})$. When one of the phenyl group is dissociated from CV, two large fragments of $120 \mathrm{amu}$ and $252 \mathrm{amu}$ are formed schematically. The prominent mass peak at $270 \mathrm{amu}$ is observed in the mass spectrum. This large fragment of $270 \mathrm{amu}$ suggests that the one of the phenyl ring cleaves to form relatively smaller fragments $(<70 \mathrm{amu})$ compared to aniline ring. The detail of smaller mass region $(<100 \mathrm{amu})$ shown in Fig. 4 suggests that the mass peaks observed in this region consist not of tita- 


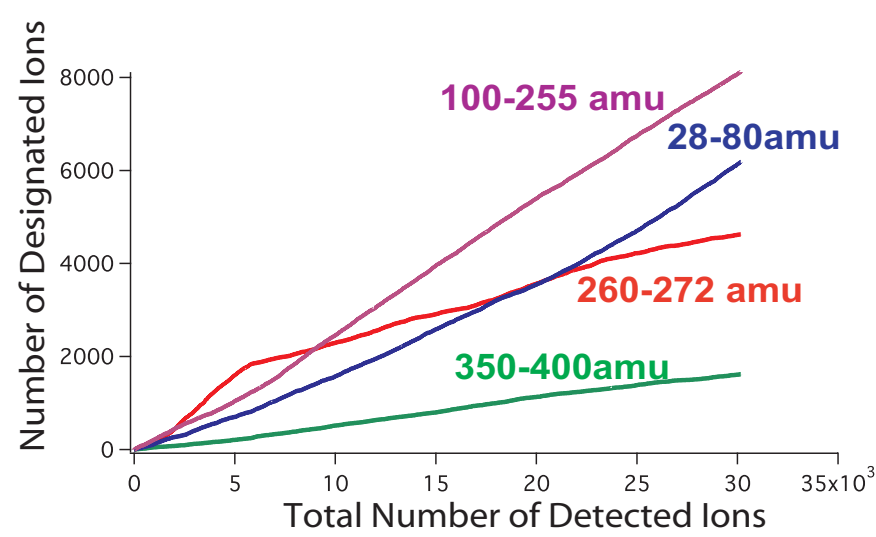

FIG. 5: The ladder diagram of CV ions detected from CV supported on Ti tip by using 3D-SAP triggered by 3rd harmonics of $\mathrm{Nd}: \mathrm{YVO}_{4}$.

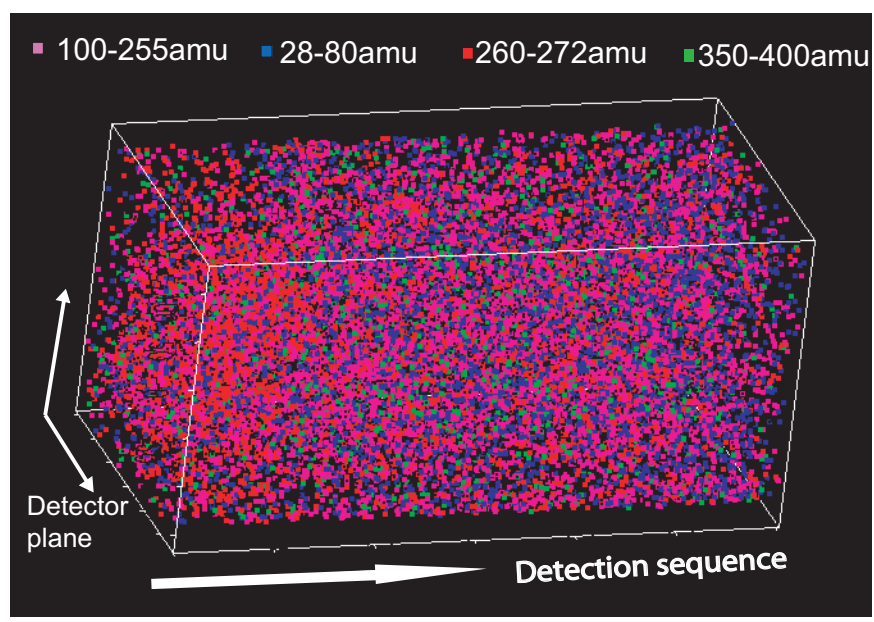

FIG. 6: The distribution of CV ions detected from CV coated on Ti tip by using 3D-SAP triggered by 3rd harmonics of $\mathrm{Nd}: \mathrm{YVO}_{4}$. The analysis proceeded from left to right along the horizontal axis. The colors of ions (dots) correspond to the designated mass peak in Fig. 3 and the ladder diagram.

nium cations but of organic cations due to their peak-peak seperation $(1 \mathrm{amu})$.

The ladder diagram of $\mathrm{CV} / \mathrm{Ti}$ tip of Fig. 5 shows that the large fragments of CV molecule are dominant in the early stage up to $5000 \mathrm{cnts}$. The growth rate of the designated ions are constant respectively after $5000 \mathrm{cnts}$. The 3D-distribution of Fig. 6 shows that the abundance of fragments of 260 272 amu is higher in the surface region corresponding to the ladder diagram. The ion distribution on the detector surface is almost homogeneous as in the result of bare Ti tip analysis shown in Fig. 2.

When the same molecular system supported on metal substrate is AP analyzed by applying voltage pulse, it shows different behavior. Figure 7 shows the mass spectrum obtained for CV supported on Ti tip by using voltage pulse in $\mathrm{AP}$ analysis. The mass peak of $\mathrm{CV}$ fragment has a significant tailing in large mass side. The ions ascribed to titanium are not observed as distinct mass peak though the noise level ("grass") is not low enough. The poor mass resolution is partly due to energy dispersion in linear time of flight (ToF) mass analysis.

Figure 8 shows the distribution of ions of the same data

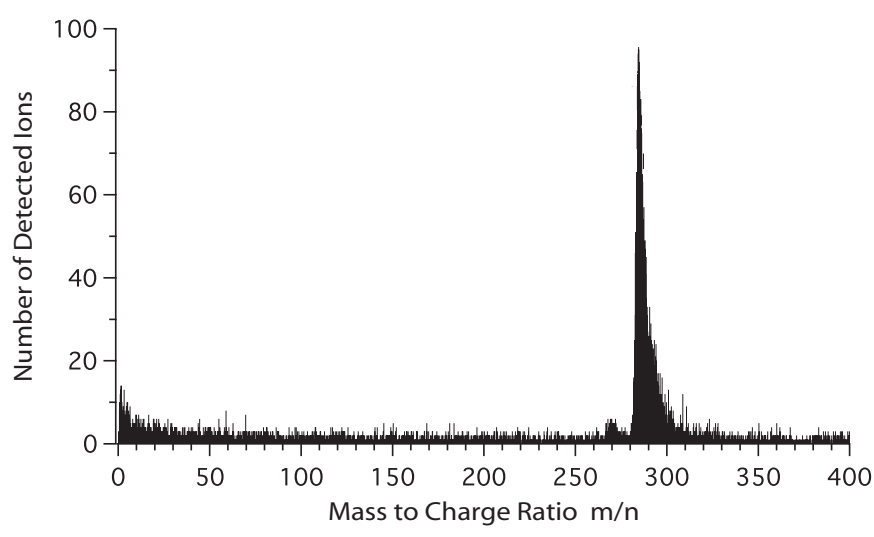

FIG. 7: The mass spectrum of $\mathrm{CV}$ ions detected from $\mathrm{CV}$ supported on Ti tip by using 3D-SAP triggered by voltage pulse.

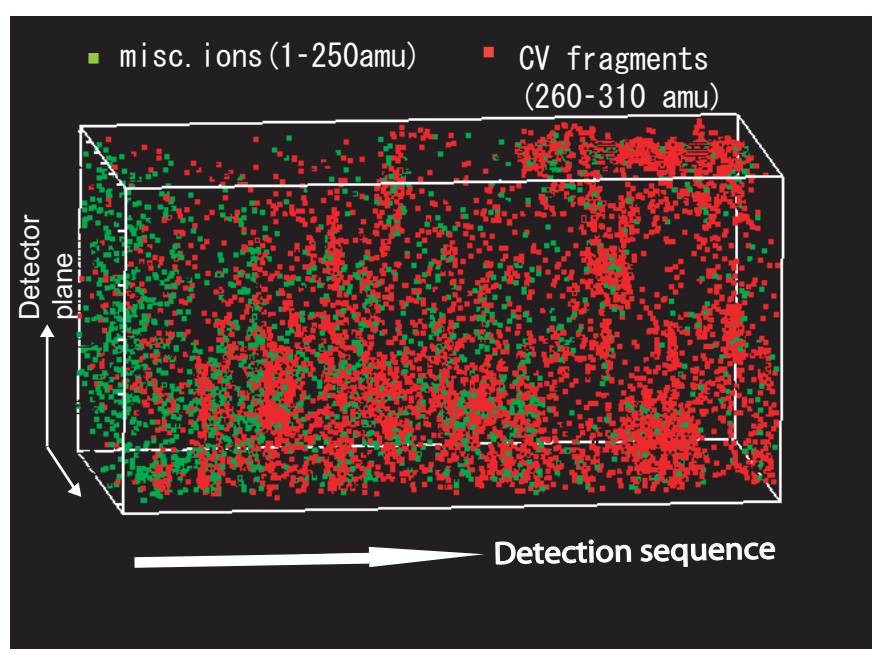

FIG. 8: The distribution of CV ions detected from CV supported on Ti tip by using 3D-SAP triggered by voltage pulse. The analysis proceeded from left to right along the horizontal axis. The CV fragment ions are plotted using red color.

of Fig. 7. The ions are gathering in some region and not distributed homogeneously in the detection plane therefore the 3D-plot using detection order as Z-axis shows a lot of hollow or empty region in the data space.

The change of supporting material from titanium to tungsten caused some change in mass spectrum. Figure 9 shows the mass spectrum of CV supported on tungsten by using voltage pulse for triggering. The spectrum shows that the almost the same large fragment mass peak of $\mathrm{CV}$ and the relatively sharper peak in the smaller mass region $(<100 \mathrm{amu})$. The mass peaks around $50 \mathrm{amu}$ can be assigned not to tungsten ion $\left(\mathrm{W}^{3+}, \mathrm{W}^{4+}\right.$, etc.) but to CV fragments because the peak width of tungsten has to be broader than that of the observed one when the mass resolution is not good enough for separating 4 major isotopes of tungsten.

The ladder diagram shown in Fig. 10 indicates that the ions of smaller mass appeared in the initial part of the analysis and the large mass fragments are dominant in the latter part. Figure 11 shows the distribution of the ions in this data. The ions are not covering the whole surface of the detector also as in the case of CV supported 


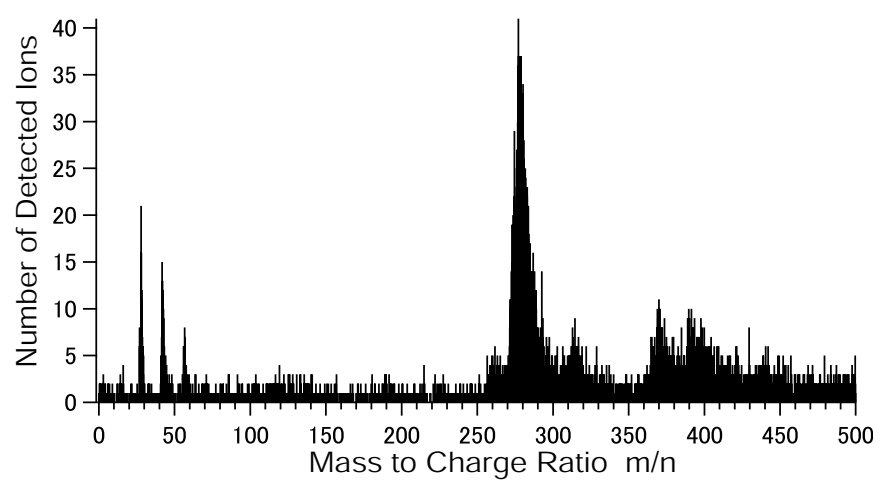

FIG. 9: The mass spectrum of $\mathrm{CV}$ ions detected from $\mathrm{CV}$ supported on $\mathrm{W}$ tip by using 3D-SAP triggered by voltage pulse.

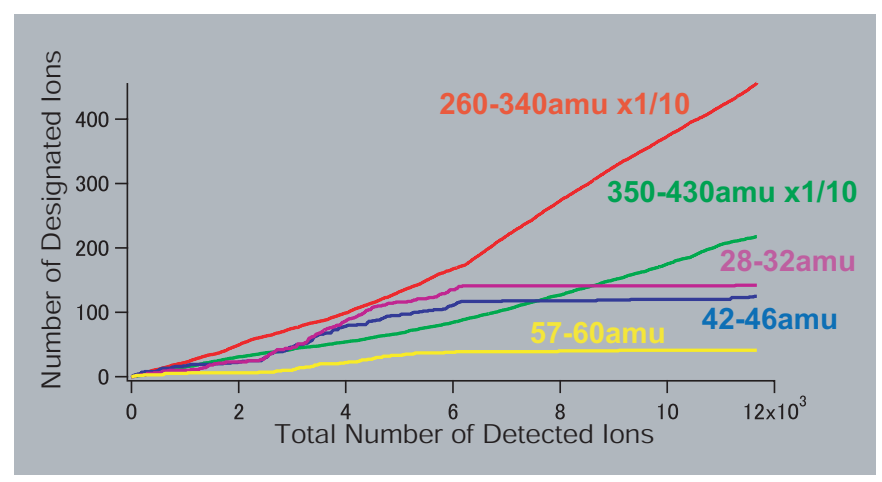

FIG. 10: The ladder diagram of $\mathrm{CV}$ ions detected from $\mathrm{CV}$ supported on $\mathrm{W}$ tip by using 3D-SAP triggered by voltage. The counts of dominant ions of larger mass region $(>250 \mathrm{amu})$ are reduced to $1 / 10$.

on Ti tip. Though the compositions of ions are slightly different, especially in the low mass region, the distribution anomaly of ions is observed in the both cases. The difference of the mass spectrum might be coming from the difference of catalytic effect of the substrate materials.

The ion distribution observed in voltage pulse AP shows anomaly as shown in Fig. 8 and Fig. 11. The presence of "hot" region suggests that there is reaction process which is enhanced by specific site on the tip apex region though the migration of the molecular species (reactant) is not enhanced by voltage pulsing. Though the poor mass resolution of the voltage pulsing AP compared to that of laser pulsing AP is consistent with common understanding, the distribution contrast of voltage pulsing AP seems to carry some information compared to flat distribution in laser pulsing AP.

The distribution of fragment ions suggests that the molecular species diffuse from shank or neighboring region to the tip apex to evaporate after ionization. The strong electric field and the diffusion of mobile phase material are also coupled in charged droplet formation which is used in liquid metal ion source (LMIS), electro-spray ionization (ESI), etc.

The laser pulsing causes the temperature rise at the tip apex in the order of several hundred Kelvin [6] in contrast to the small temperature effect of voltage pulsing. There is no doubt about enhancement of surface migration due

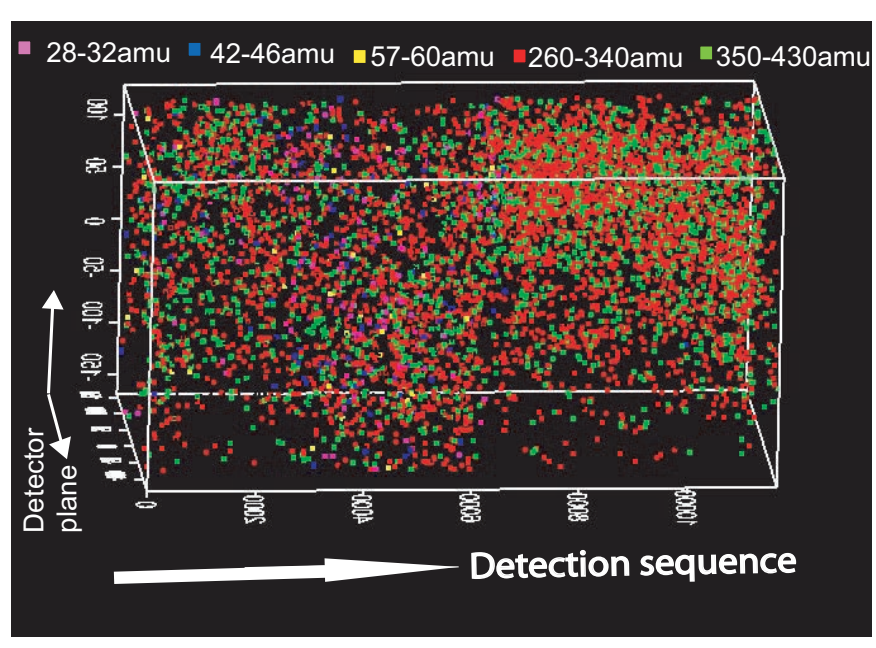

FIG. 11: The distribution of CV ions detected from CV supported on $\mathrm{W}$ tip by using 3D-SAP triggered by voltage pulse. The analysis proceeded from left to right along the horizontal axis.

to laser irradiation, the flat distribution of fragment ion shows that the ionization/fragmentation is not dependent on specific sit in laser pulsing AP.

The raw ion hit data collected by using 2-dimensional ion detector is generally transformed in $3 \mathrm{D}$ atom probe tomography by using the parameters of applied bias voltage and the tip apex curvature to obtain the real space $\mathrm{X}, \mathrm{Y}$, and $\mathrm{Z}$ coordinates of ions $[5]$. The evaporation field is determined by the tip curvature and the bias voltage. The tip curvature is directly related to the magnification ratio and the field of view in AP. Therefore, the raw hit position on the detector is re-scaled by the change of applied voltage keeping the evaporation rate constant in AP analysis. The detection order of ions is used for their $\mathrm{Z}$ coordinates in $3 \mathrm{D}$ plot with adjustment to reproduce the density of the material. This reconstruction procedure is justified by numerous publication for solid state material such as metals and semiconductors kept at cryogenic temperature. In this reconstruction treatment, the spatial densities of the detected species are assumed to be constant inside the sample (matrix) material. The migration of the species can be suppressed by cooling down the sample temperature.

But the results presented here does not fit with the same treatment because the molecular systems measured here are not bulk materials and the hit positions of ions do not show the relative position in their solid structure though the angular distribution of organic ions may reflect their relative configuration just before their desorption in the apex region of the support material. Therefore the ion hit data don't match with the transformation to real space coordinates. Some other procedure is demanded to extract the ion-ion correlation information during the surface reaction promoted by field ionization/evaporation process.

The authors proposed the ion-ion coherence analysis of the AP data set [8]. The ion-ion relation during the field evaporation sequence could be extracted by coherence check from the AP data set. To make the coherence analysis more reliable, the collection of the ions in the 
wider angular part is desired by using the 3D-AP working with larger solid acceptance angle.

\section{Acknowledgments}

This work was supported by Grant-in-Aid for Creative Scientific Research No.18GS0204 from Japan Society for the Promotion of Science.
[1] M. K. Miller, A. Cerezo, M. G. Hetherington, and G. D. W. Smith, Atom Probe Field Ion Microscopy (Oxford Science/Clarendon Press, New York, 1996)

[2] D. Blabvette, A. Bostel, J. Sarrau, B. Deconihout, and A. Menand, Nature (London) 363, 432 (1993).

[3] O. Nishikawa, M. Kimoto, M. Iwatsuki, and Y. Ishikawa, J. Vac. Sci. Technol. B 13, 599 (1995).

[4] O. Nishikawa, M. Watanabe, T. Murakami, T. Yagyu, and M. Taniguchi, New Diam. Front. Carbon Technol. 13, 257 (2003).
[5] P. Bas, A. Bostel, B. Deconihout, and D. Blavette, Appl. Surf. Sci. 87 / 88, 298 (1995)

[6] F. Vurpillot, J. Houard, A. Vella, and B. Deconihout, J. Phys. D: Appl. Phys. 42, 125502 (2009).

[7] G. Taylor, Proc. R. Soc. London, Ser. A 280, 383 (1964).

[8] M. Taniguchi, O. Nishikawa, S. Komata, S. Watanabe, A. Yamagishi, and T. Sasaki, e-J. Surf. Sci. Nanotech. 4, 1 (2006). 